Revista Calidad en la Educación Superior

Programa de Autoevaluación Académica

Universidad Estatal a Distancia, Costa Rica

ISSN 1659-4703

revistacalidad@uned.ac.cr

\title{
INNOVACIÓN Y CALIDAD EN LA EDUCACIÓN NO FORMAL: EDUCACIÓN TÉCNICA TIC DESDE LA REGIÓN PACÍFICO CENTRAL
}

\author{
INNOVATION AND QUALITY IN NON-FORMAL EDUCATION: TECHNICAL \\ EDUCATION ICT FROM THE CENTRAL PACIFIC REGION
}

\author{
Grethel Mena Araya ${ }^{1}$, grmena@uned.ac.cr \\ Cecilia Barrantes Ramírez ${ }^{2}$, cbarrantes@uned.ac.cr \\ Universidad Estatal a Distancia, Costa Rica
}

\author{
Volumen 6, número 1 \\ Mayo 2015 \\ Pp. $150-173$
}

Recibido: 21 enero, 2015

Aprobado: 14 abril, 2015

\footnotetext{
1 Grethel Mena Araya, Ingeniera en Informática, Master en Administración Educativa de la UNED; a partir del 2001-2007, Encargada de la Cátedra de Desarrollo de Sistemas, carrera de Informática Administrativa de la ECEN; 2007-2014 Coordinadora Área de Comunicación y Tecnología de la Dirección de Extensión; Lideré por parte de la UNED el proyecto de regionalización SIR-PC desde el 2009 ; Coordinadora del Proyecto de Gestión e Innovación en TIC; a partir del 2012-2014; desarrollando un programa de Educación no Formal en TIC en Telemática y Seguridad Informática para el Pacífico Central con los PROYECTOS IIIDR-PC-CONARE.

2 Cecilia Barrantes Ramírez, Directora de Relaciones Internacionales y Cooperación, UNED. Ingeniera Industrial, Gerente de Proyectos del Programa de Relaciones Exteriores, UNED 2005- 2011. Miembro de Nexo, CONARE; Sistema de Universidad y Sociedad y Sistema Internacionalización, CSUCA. Responsable académico, docente y evaluadora de la Escuela de Ciencias de la Administración UNED por 10 años, profesor de Ingeniería Industrial en la Universidad Internacional de las Américas, profesor de la Organización Internacional del Trabajo (OIT), el Instituto Nacional de Aprendizaje (INA), Centro para la Administración pública Investigación y Formación (CICAP), CAPRIS SA, Cooperativa Dos Pinos y otros 1999-2001.
} 


\title{
Resumen
}

Las tendencias nacionales e internacionales en la educación técnica se han convertido en una poderosa herramienta para que muchas poblaciones mejoren sus capacidades y conocimientos en el tema de las tecnologías y aseguren mejores oportunidades para acceder al mercado laboral, e incluso que desarrollen su propio emprendimiento.

Esta tendencia ha sido promovida por organismos internacionales como la ONU, así como por empresas nacionales que, por medio de sus cámaras, han propiciado la búsqueda de oportunidades de capacitación para los y las jóvenes de diferentes regiones, una de ellas es la Cámara de Tecnologías de Información y Comunicación (CAMTIC).

El área de comunicación y tecnología de la Dirección de Extensión en la UNED ha dado respuesta a este llamado ofreciendo cursos y programas que preparan a la población para enfrentar ese cambio tecnológico que las empresas viven día a día y que les hace requerir personal calificado.

Estas iniciativas, en especial la del Técnico en Telemática y Seguridad Informática - desarrollado en la región del Pacífico Central-, han tenido una gran acogida por la población, que no solo considera el proyecto como una oportunidad para su desarrollo, sino también para el mejoramiento de las condiciones de vida de la región.

Palabras claves: educación técnica, emprendedurismo, educación continua

\begin{abstract}
National and international trends in technical education has become a powerful tool for many people improve their skills and knowledge in the field of technology and ensure better opportunities to enter the labor market and even develop their own business.

This trend has been promoted by international organizations like the ONU, as well as national companies through their Chambers has led the search for training opportunities for young people from different regions, one of which, the Chamber of Information and Communication Technologies (CAMTIC).

The area of communication and technology form the Extension Department at UNED has responded to this call by offering courses and programs that prepare people to face this technological change that companies live day to day and that makes them require qualified personnel.

These initiatives, in particular the Technical Telematics and Security, developed in the Pacific Region Central, have been welcomed by the population that considers not only the project as an opportunity for development but also for the improvement conditions of life in the region.
\end{abstract}

Keywords: Technical education, entrepreneurship, continuing education 
Como parte de la educación superior se contempla la educación técnica como aquella educación orientada a ofrecerles a los y las estudiantes conocimientos y capacidades para que puedan desempeñarse en alguna especialidad o por su propia cuenta, es decir, brindarles herramientas para fomentar el emprendedurismo.

La educación técnica y la formación profesional no tienen solo el objetivo de preparar a la población joven para el empleo, como los otros tipos de enseñanza. Deben contribuir también a la preparación de las personas jóvenes para la vida ciudadana, su desarrollo cultural y político y su vida privada. (Estado de la Nación, 2003, p. 237).

A nivel internacional, la educación y la formación técnico-profesionales han ido evolucionando a través del tiempo, ajustándose a las transformaciones y nuevas realidades productivas. En sus orígenes la formación profesional fue concebida como un sistema exclusivo para trabajadores y trabajadoras, caracterizado por su flexibilidad, su carácter terminal y su independencia del sistema educativo regular. Sin embargo, esta concepción se modificó debido a la influencia de los grandes avances tecnológicos, científicos y económicos experimentados en el siglo XX. La velocidad de las innovaciones de la ciencia y la tecnología, y los consecuentes cambios en los mercados laborales, han generado una conciencia más clara sobre la necesidad de que las personas no solo se formen en una especialidad técnica, sino que además se preparen para el aprendizaje continuo y la posible rotación laboral entre diferentes áreas técnicas e incluso entre sectores productivos (Estado de la Nación, 2003, p.237)

La educación técnica les permitirá a las y los jóvenes aprovechar las oportunidades de empleo y de desarrollo empresarial mediante un conjunto de aptitudes, competencias y destrezas que los habilitarán y les facilitarán su inserción en el mundo laboral, el aprendizaje a lo largo de la vida y el desarrollo a 
fin de que sean personas plenas y dignas; estas figuran entre las aspiraciones nacionales propuestas en el informe del Estado de la Nación (2003).

Según la Coalición Costarricense de Iniciativas de Desarrollo (CINDE), hoy Costa Rica es muy diferente a lo que fue años atrás, es un país que compite en un mundo globalizado donde la tecnología es protagonista. Esta realidad ha cambiado en gran medida la vida y el mercado, ya que aquellas carreras que tradicionalmente gozaban de gran atractivo han dado paso a nuevas especialidades técnicas y universitarias relacionadas con la ciencia, las matemáticas y la tecnología. Los técnicos son hoy un recurso de gran importancia y altamente cotizado por las empresas.

Por su parte, la Cámara de Tecnologías de Información y Comunicación (CAMTIC) ha recalcado que Costa Rica tiene la necesidad de aumentar la cantidad de personal con conocimientos especializados en ciencia y tecnologías digitales, debido al crecimiento que ha logrado en la generación de productos y servicios, investigación, desarrollo e innovación en estas áreas.

La velocidad de las innovaciones en la ciencia y la tecnología y los consecuentes cambios en los mercados laborales ha generado una conciencia más clara sobre la necesidad de que las personas no solo se formen en una especialidad técnica sino que además se preparen para el aprendizaje continuo y la posible rotación laboral entre diferentes áreas técnicas e incluso entre sectores productivos (Estado de la Nación, 2003, p.238).

Se sabe que «la innovación es la selección, organización y utilización creativas de recursos humanos y materiales de maneras nuevas y propias que den como resultado la conquista de un nivel más alto con respecto a las metas y objetivos previamente marcados» (Estado de la Nación, 2003). Estas innovaciones 
en ciencia y tecnología hacen necesario que las universidades desarrollen programas igualmente innovadores para la enseñanza de estas nuevas herramientas y contribuir con el desarrollo de las comunidades. La Universidad Estatal a Distancia, a través del área de Comunicación y Tecnología de la Dirección de Extensión, ha enfrentado este reto, conociendo la gran demanda de las carreras de Tecnologías de Información y Comunicación y la necesidad de impactar en diferentes zonas del país con carreras técnicas, de manera que ha desarrollado programas y cursos especializados en tecnologías que le proporcionen a las personas de diferentes comunidades del país herramientas para la generación de sus propias empresas, o bien para alcanzar con éxito puestos de trabajo en instituciones que requieren de estas especialidades. Un ejemplo de esto es el Técnico en Telemática y Seguridad Informática (TTSI) implementado en la zona del Pacífico Central. A continuación una descripción de la experiencia resultante de la implementación del programa de educación no formal técnica en la Región Pacífico Central.

\section{Caso de éxito desarrollado por la UNED}

Durante tres años, por medio de Programa de Regionalización Interuniversitaria de CONARE, mediante las iniciativas interuniversitarias de regionalización del Pacífico Central "IDR_PC" se han estado impactando las regiones del pacifico central con un proyecto llamado Técnico en Telemática y Seguridad Informática desarrollado por la UNED, el cual está destinado a capacitar a las poblaciones más vulnerables de la región periférica del Pacífico Central, entre ellas Monteverde, Orotina, Puntarenas, Miramar, Esparza, Quepos y Jacó.

El propósito del técnico ha sido mejorar las capacidades personales y laborales de los y las estudiantes que puedan ingresar a dicho proyecto. El programa académico se impartió específicamente en cinco sitios en los que existe afluencia de la población meta con el perfil requerido. Desde la escogencia de las personas 
se está aportando a la calidad académica, en el sentido de contar con el perfil deseado para que se pueda alcanzar la meta propuesta.

El objetivo principal es fortalecer la educación no formal técnica ofreciendo una capacitación técnica en Telemática y Seguridad Informática dirigido a personas que buscan adquirir conocimiento en las TIC, especializándose en el área de redes informáticas. Este proyecto se fundamenta en la educación técnica por medio de un programa académico respaldado por la Academia de Innovación Tecnológica UNED-CISCO, cuyo objetivo principal es formar personas con conocimiento, competencias y habilidades en esta área.

Este tipo de educación no solo resulta beneficioso para estas poblaciones, sino que también contribuye con el desarrollo de la región y del país. Por ende, mejora la calidad de vida de estas personas que adquieren más oportunidades de empleo e ingresos y la posibilidad de continuar su proceso formativo con éxito.

El programa brinda cursos de emprendedurismo, como Informática Aplicada a los Negocios, ya que el proyecto pretende fomentar en los y las estudiantes emprendimientos para MyPymes basados en TIC como pequeños empresarios en soporte técnico e instalación de redes informáticas, ya que la región requiere una mayor flexibilidad en los diferentes procesos, según su enfoque de mercado. Esto permite mayor productividad, eficiencia y eficacia, para hacerlos más competitivos en el mercado nacional.

Es importante mencionar que este proyecto ha tenido gran impacto en las personas beneficiadas, debido a que pueden incursionar en empresas importantes de desarrollo tecnológico, tanto a nivel nacional como transnacional.

El área académica que respalda el proyecto por parte de la UNED es el Área de Comunicación y Tecnología de la Dirección de Extensión; el recurso académico 
ha sido aportado por esta área y se basa en ingenieros e ingenieras en Computación e Informática con amplia experiencia profesional y talento humano, personas comprometidas con la formación académica de los y las estudiantes que se encuentran dentro de este programa.

En el proyecto de telemática se desarrolló un plan de estudio riguroso y de calidad, tanto presencial como virtual, con el propósito de cumplir con las metas establecidas; por otra parte, se dio seguimiento al proceso de formación, al rendimiento académico de la población meta dentro del tiempo planeado y a la ejecución del proyecto.

Parte de la calidad del proceso de enseñanza-aprendizaje de los y las estudiantes fue llevar a cabo visitas de seguimiento a cada centro universitario o lugar donde realizaron la primera etapa del proyecto, esto con el propósito de darles un acompañamiento hasta que desarrollaran sus emprendimientos.

Dentro de las metas propuestas en este proyecto para alcanzar la calidad en la educación no formal, en este proyecto específico de TTSI se planteó lo siguiente:

- Mantener el interés y evitar la deserción de los y las estudiantes, incentivando la comunicación y las mejoras continuas en las técnicas de aprendizaje para cada estudiante de forma individual, mientras demuestren un interés real por cumplir con la meta.

- Ofrecer las certificaciones CISCO CCNA como complemento en el TTSI, como una oportunidad de mejora en la generación de nuevas tendencias en las TIC; esto le permite al estudiante estar más motivado y comprometido con la iniciativa.

- Obtener retroalimentación con el propósito de conocer la satisfacción de la población en cuanto a la capacitación que está recibiendo y mejorar el proceso. 
- Realizar visitas de seguimiento, llevadas a cabo con el propósito de obtener una retroalimentación entre los y las estudiantes, la persona coordinadora y los tutores y las tutoras del proyecto.

Este proyecto se desarrolló en dos etapas: en la primera etapa participaron los centros universitarios de Orotina, Puntarenas y Monteverde, y para la segunda etapa participaron los centros de Quepos, Puntarenas y la comunidad de Jacó.

En la primera etapa se capacitaron 79 personas en total entre las regiones de Monteverde, Orotina y Puntarenas, lo cual representa un número significativo sobre el impacto que se puede obtener de este proyecto en cuanto al mejoramiento de la calidad de vida de las poblaciones.

Para la segunda etapa se contó con alrededor de 45 personas, para un total de 124 estudiantes, que terminaron en noviembre de 2014.

El impacto de los proyectos en la región Pacífico Central ha sido positivo, apoyando el desarrollo regional de las personas emprendedoras que buscan oportunidades para introducir sus ideas productivas en el mercado. Se ha logrado contar con el apoyo de profesionales certificados como instructores CISCO para la ejecución del proyecto.

El trabajo de campo que se llevó a cabo, entre el año 2012-2014, mediante la Iniciativa interuniversitaria de regionalización del Pacífico Central (IIDR-PC), por medio del proyecto de Gestión e innovación en TIC arrojó algunos resultados de las siguientes experiencias en los Centros universitarios involucrados, las cuales fueron producto las visitas de seguimiento, entrevistas, evaluaciones por parte de los estudiantes de cada uno de los cursos que se impartieron. CONARE (2014). 


\section{Experiencia en Monteverde}

En cuanto al impacto que tuvo el proyecto en un año y medio con respecto a los y las estudiantes de la zona de Monteverde, podemos mencionar, según lo muestra el gráfico 1, que iniciaron en el programa en primera instancia doce personas matriculadas; se aprecia que aprobaron el módulo 1 todos los estudiantes que matricularon, y finalizando el módulo 2 se observa que hubo disminución de dos estudiantes que no aprobaron. $\mathrm{Y}$ para el módulo 3 se aprecia que solo ocho estudiantes terminaron, uno desertó y otro reprobó, ya en el módulo 4 se mantienen ocho estudiantes de doce que iniciaron, estos ocho llegan al final para graduarse.

Gráfico 1. Resultado por módulo en Monteverde

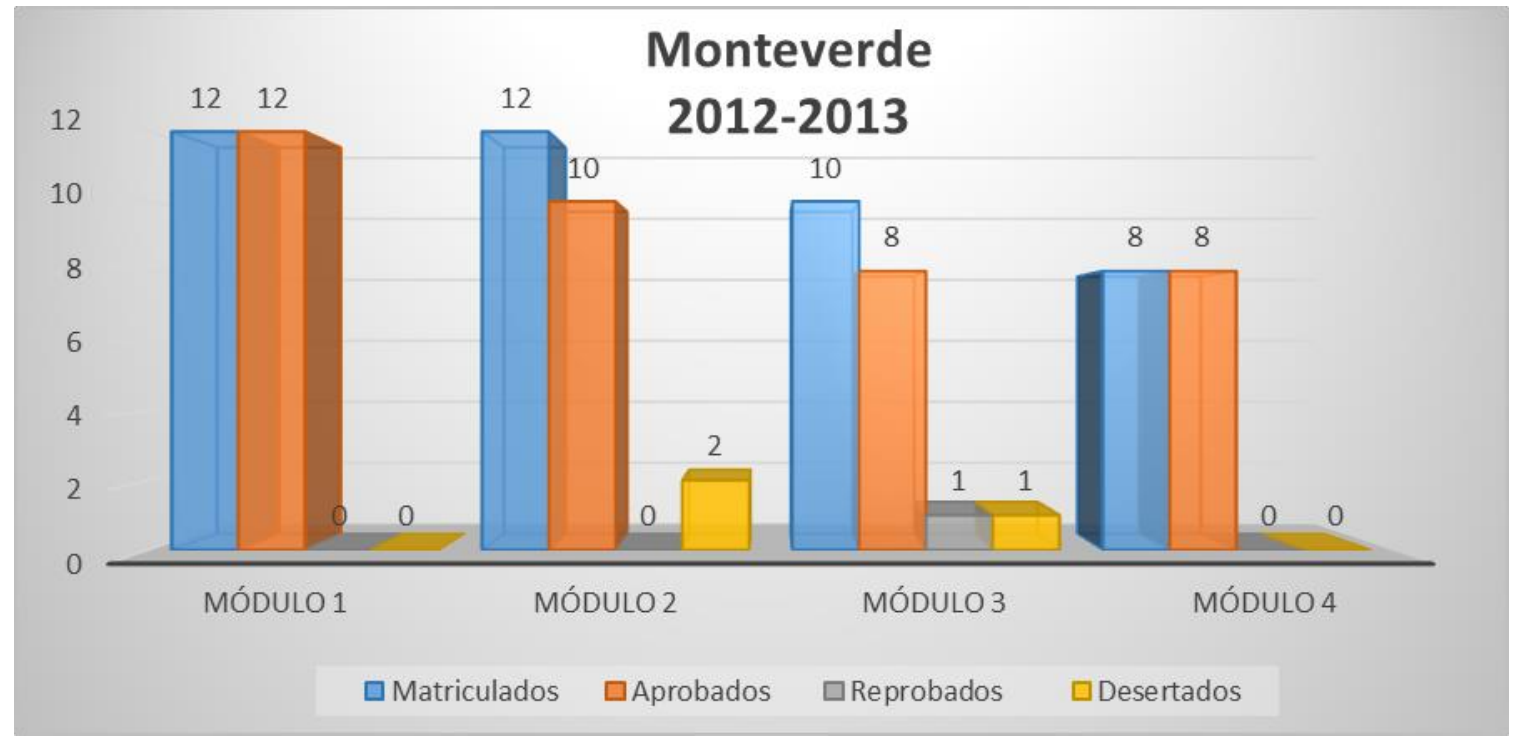

Fuente: Elaboración propia

Es importante mencionar que ellos solo tienen educación media hasta noveno año, además nunca habían tenido la oportunidad de cursar un programa académico de tipo tecnológico.

Las y los estudiantes interesados en la iniciativa del Técnico en Telemática y Seguridad Informática (TTSI) se han comprometido, lo que ha generado un gran interés y perseverancia de algunos y algunas estudiantes por los conocimientos 
adquiridos y las evaluaciones sumativas y formativas que se reflejan en diferentes actividades, como proyectos y trabajos cotidianos a corto o mediano plazo.

Al hablar del impacto del proyecto se denota un entorno de satisfacción por parte de los y las estudiantes, puesto que todas las personas que iniciaron se han mantenido hasta el momento, y más bien el interés ha hecho que ellas mismas den a conocer los beneficios que se pueden obtener a corto o mediano plazo con la iniciativa, la cual puede ser una fuente primaria de generación de empleo en estas zonas del Pacífico Central. Invertir en esta iniciativa es dar un incentivo a personas de escasos recursos con situaciones socioeconómicas difíciles. Ayudan no solo al estudiante como beneficiado directo, sino también a las personas que lo rodean como beneficiadas indirectas, llámense hijos e hijas, madres y padres 0 aún hermanos y hermanas.

Por otra parte, los logros han venido a ser la base para conocer lo que la comunidad necesita; sin embargo, también existen los aspectos débiles que se deben fortalecer, como los que se mencionan a continuación:

- En estas zonas no existen empresas de soporte técnico, por lo que es necesario brindar los conocimientos básicos para la reparación y el mantenimiento de equipos computacionales y para dar las bases sobre prevención y corrección de problemas en los y las estudiantes, y que con esto puedan ayudar a solventar las necesidades de la comunidad y al mismo tiempo generar algún tipo de ingreso extra.

- En el caso de Puntarenas y Monteverde, estas son zonas cuya principal actividad económica es el turismo.

- Es necesario generar en el o la estudiante el quehacer autodidacta para la resolución de problemas técnicos y operativos en la implementación de redes para el hogar y pequeñas empresas.

- A pesar de que el estudiantado ha logrado conocer diferentes parámetros para establecer un criterio técnico y basar su criterio en los conocimientos 
adquiridos mediante el TTSI, es importante reforzar más los conocimientos y fortalecer el nivel práctico para establecer la factibilidad de llevar a cabo proyectos reales de reparación, mantenimiento o adquisición de equipo computacional para redes.

Ya en este nivel las y los estudiantes son capaces de conocer y reconocer el tipo de cableado necesario para cumplir las necesidades físicas de una infraestructura de red solicitada por algún cliente. Todos estos logros son un resumen de las evidencias de la población estudiantil en las diferentes tutorías, proyectos prácticos y laboratorios dirigidos que se han llevado a cabo, donde han externado ejemplos vinculados al lugar de trabajo o situaciones a las que en ocasiones se enfrentan fuera de la academia.

Se pretende mantener el interés y evitar la deserción de las y los estudiantes, incentivando la comunicación y la mejora continua de las técnicas de aprendizaje para cada estudiante de forma individual, mientras demuestren un interés real por cumplir con la meta.

\section{Experiencia en Puntarenas centro}

Con respecto a la población estudiantil de la zona de Puntarenas centro, en el desarrollo de los cursos se ha notado el avance en conocimiento y destrezas que los y las estudiantes han adquirido durante su proceso de aprendizaje; los objetivos de cada uno de los cursos se han revisado al finalizar, comprobándose su cumplimiento. De forma generalizada la población estudiantil ha externado su satisfacción al concluir cada proceso.

Se aprecia que en el CU de Puntarenas hubo dos grupos en el mismo periodo. Como puede observarse en los gráficos 2 y 3 , en el grupo 1 iniciaron veinticuatro estudiantes, sin embargo en el módulo 1 solo ganaron el curso catorce estudiantes y hubo una deserción de diez estudiantes; ya en el módulo 2 aprobaron solo trece 
personas y hubo un estudiante que reprobó el modulo, y no hubo deserción. Igualmente se puede ver que en el módulo 3 siguieron los mismos trece estudiantes, sin embargo desertó uno, y se culminó en el módulo 4 con doce estudiantes de veinticuatro, que fueron los que se graduaron al final.

\section{Gráfico 2. Resultados por módulo en Puntarenas, grupo 1}



Fuente: Elaboración propia

\section{Gráfico 3. Resultados por módulo en Puntarenas, grupo 2}

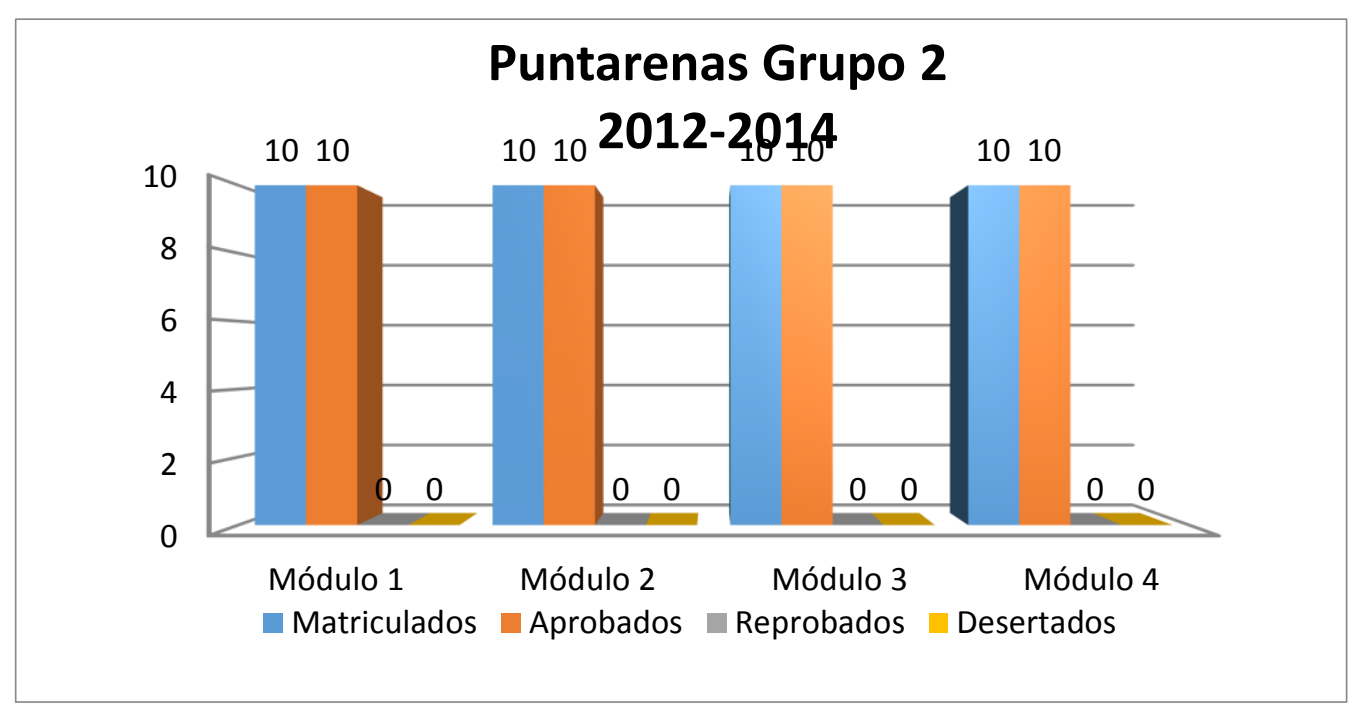

Fuente: Elaboración propia 
Con respecto a los y las estudiantes del grupo 2 del CU Puntarenas, al término de esta primera etapa la población estudiantil mantuvo y aumentó su interés por el proyecto e indicó que sería de gran beneficio personal y familiar. Tal como lo muestra el gráfico, iniciaron diez estudiantes y todos se mantuvieron en cada uno de los módulos, al final culminaron todos y se graduaron.

\section{Experiencia en Orotina}

En cuanto a los y las estudiantes de la zona de Orotina, al término de esta primera etapa mantuvieron y aumentaron su interés por el proyecto, e indicaron que sería de gran beneficio personal, familiar y para la zona de Orotina, ya que en los próximos años se estará construyendo una zona franca y un nuevo aeropuerto. En Orotina no existen, a nivel formal de empresa, servicios en tecnología, por lo que se visualiza una oportunidad en ello. Al estar tan motivados mostraron gran interés en que, al finalizar el Técnico en Telemática y Seguridad Informática, se diera continuidad con otras capacitaciones que complementaran su formación, creando en ellos ventajas competitivas.

Se aprecia en el gráfico 4 que en el CU de Orotina iniciaron dos grupos en el mismo periodo. El primer grupo inició con veintitrés estudiantes, de los cuales solo lograron aprobar diecinueve estudiantes, en tanto cuatro desertaron. En el módulo 2 hubo una baja de dos estudiantes que reprobaron, mientras diecisiete aprobaron. En el módulo 3 aprobaron diecisiete y tres desertaron, y para el módulo 4 solo se graduaron catorce estudiantes de veintitrés. 


\section{Gráfico 4. Resultados por módulo en Orotina, grupo 1}

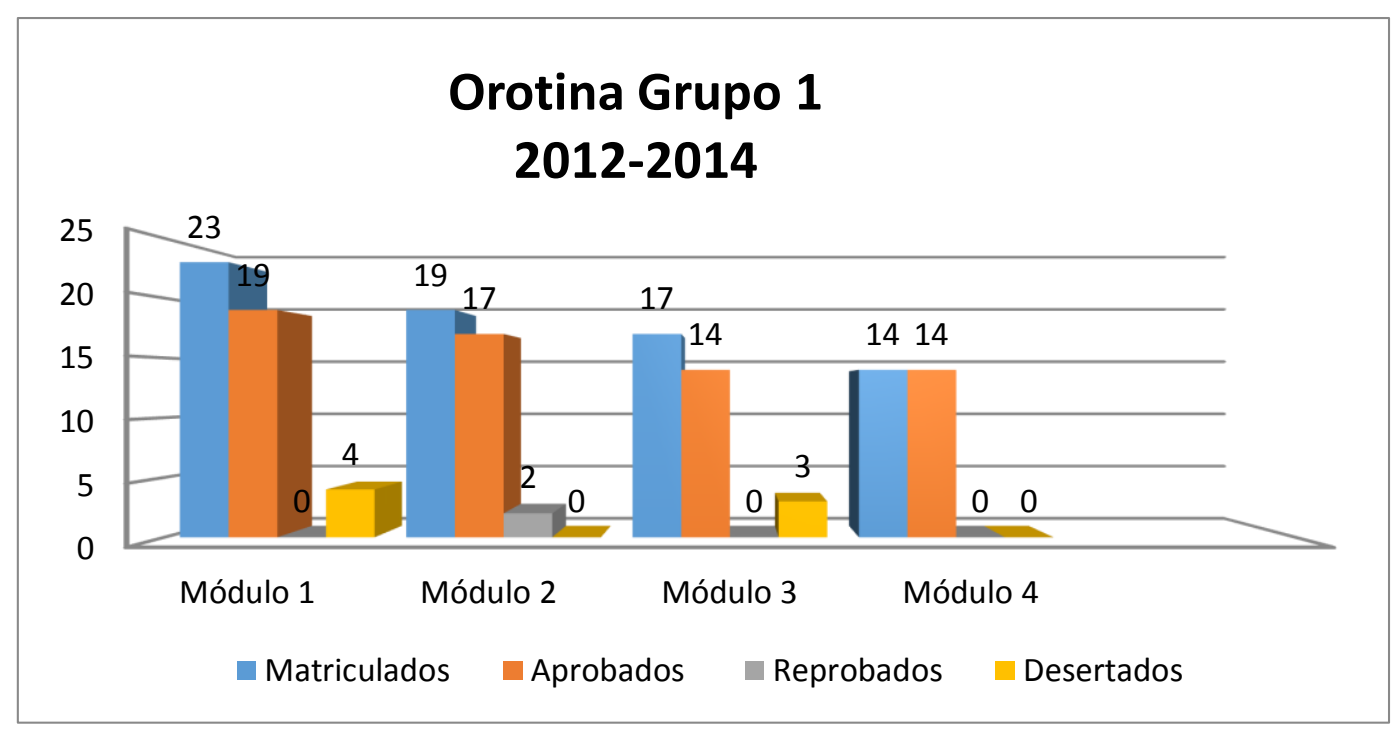

Fuente: Elaboración propia

Los y las estudiantes han logrado desarrollar destrezas de investigación, autorregulación, trabajo en equipo y trabajo colaborativo, utilizando herramientas tecnológicas que les permitieron estar en constante comunicación para lograr los objetivos planteados, pese a las distancias geográficas de cada estudiante.

En relación con los y las estudiantes del grupo 2 del CU de Orotina, según muestra el gráfico 5 , en el módulo 1 iniciaron veinte estudiantes y lograron aprobar solo dieciséis. Se aprecia desde el inicio la deserción de tres estudiantes y la reprobación de uno; ya en el módulo 2 otro estudiante reprobó, quince aprobaron el módulo 3 y para el módulo 4 se mantuvieron los quince estudiantes, que finalmente se graduaron. 


\section{Gráfico 5. Resultados por módulo en Orotina, grupo 2}

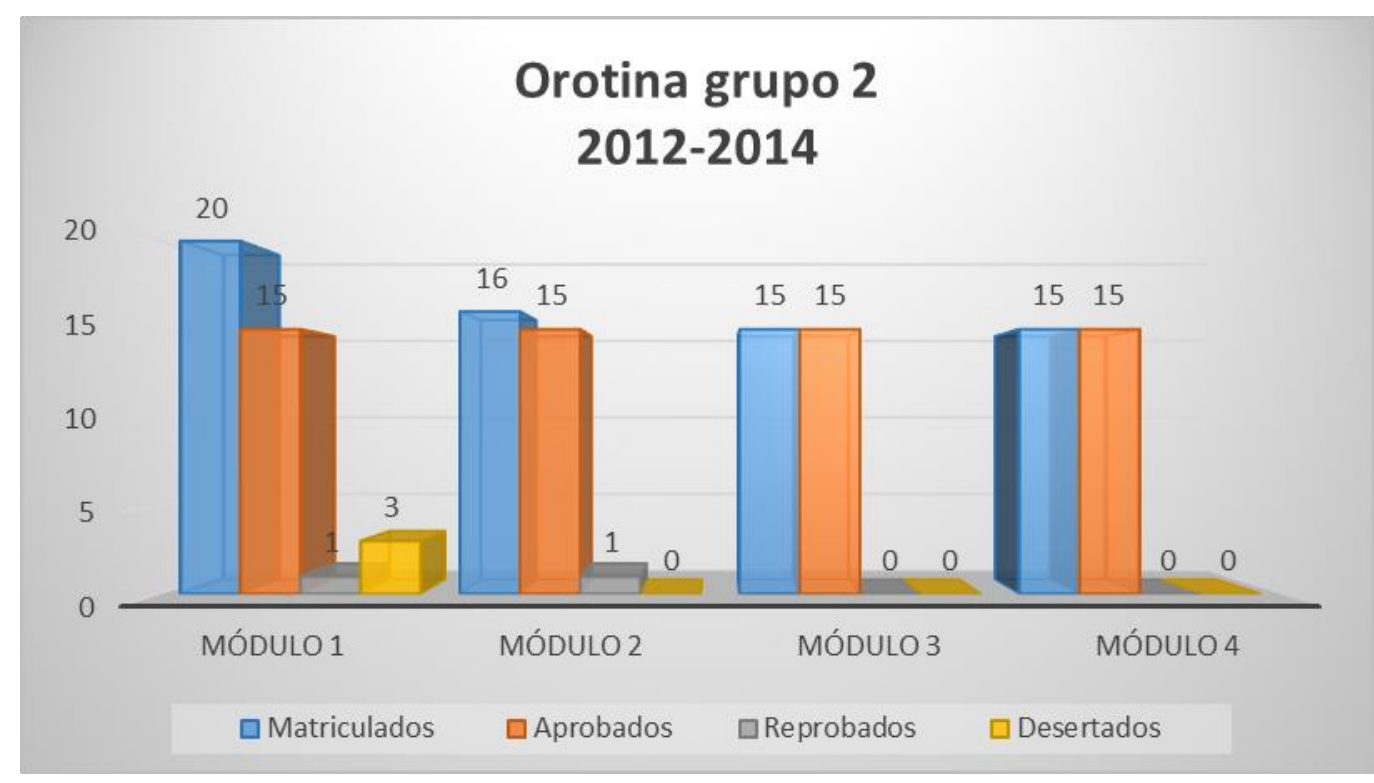

Fuente: Elaboración propia

Se ha realizado un trabajo de campo por medio de la realización de visitas a las empresas y las instituciones, analizando situaciones actuales acerca de redes informáticas, presentando recomendaciones técnicas y económicas para la solución de problemas en redes, con lo cual se aplican los conocimientos adquiridos en cursos anteriores.

Han desarrollado propuestas de proyectos en redes para una posible venta de servicios, demostrando el uso de conocimientos como electricidad, cableado estructurado, sistemas operativos, seguridad informática y trabajo de redes.

Todos los resultados obtenidos son muy satisfactorios, ya que la deserción del grupo ha sido mínima y los y las estudiantes que han salido del programa lo han hecho por problemas de tiempo en el trabajo o por temas familiares fuera del ámbito educativo, así que se puede decir que se tiene un $100 \%$ de efectividad en cuanto a la población estudiantil que gana los cursos. 
Cabe mencionar que la oportunidad que están teniendo los y las estudiantes con estos cursos fortalece de forma inmediata su nivel educativo, y lo aplicarán en las empresas donde posiblemente podrán trabajar. Estas fortalezas son las siguientes:

- Nuevos conocimientos a nivel técnico en cuanto a tecnologías, por lo que su estatus es más competitivo.

- Aumento en el nivel académico del o de la estudiante al conocer nuevos conceptos y obtener un bagaje significativo.

- Mayor oportunidad de ofertas de trabajo, ya que pueden optar por mejores puestos a nivel de TIC con el conocimiento que han adquirido.

- Mayor motivación para seguir estudiando, ya que logran obtener metas cortas y logros significativos.

- La relación estudiante-tutor y estudiante-estudiante es muy buena, ellos se esfuerzan al máximo por desarrollar y presentar de la mejor forma los trabajos y exámenes que se les encomiendan.

- La dinámica con el uso de los laboratorios es de calidad en cuanto al equipamiento de los laboratorios, ya que existen equipos en muy buenas condiciones.

\section{Experiencia en Quepos}

En el desarrollo de los cursos se ha notado el avance en conocimientos y destrezas que los y las estudiantes han adquirido durante su proceso de aprendizaje. Los objetivos de cada uno de los cursos se han revisado al final, comprobándose su cumplimiento. De forma generalizada la población estudiantil ha externado su satisfacción al concluir cada proceso.

Como se nota en el gráfico 6 del CU de Quepos, iniciaron este proceso diecinueve estudiantes, de los cuales solo quince aprobaron, dos no lograron aprobar el módulo 1 y dos desertaron. En el módulo 2 solo doce personas aprobaron, dos reprobaron y una desertó. Y para el módulo 3 solo lograron llegar once 
estudiantes, uno desertó y otro no aprobó; para culminar en el módulo 4 con once estudiantes graduados.

\section{Gráfico 6. Resultados por módulo en Quepos}

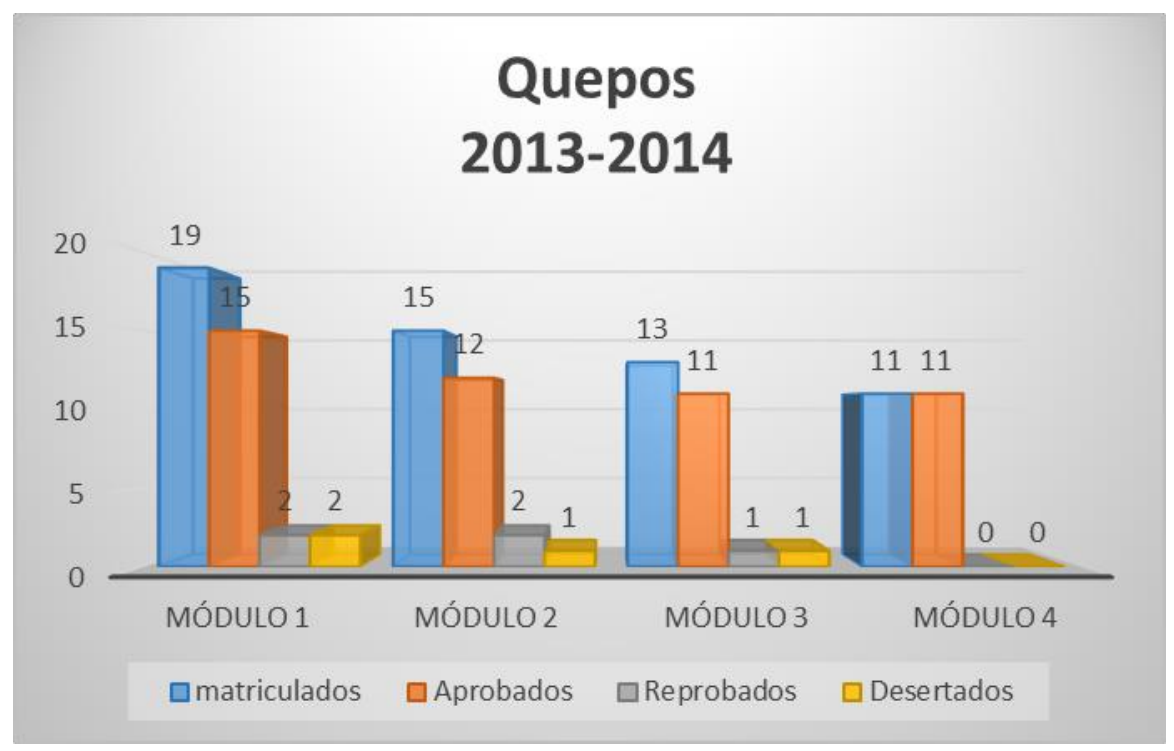

Fuente: Elaboración propia

\section{Experiencia en Jacó}

Los objetivos se han cumplido según lo especificado en el programa. Sin embargo, en la zona de Jacó no existen muchas oportunidades de trabajo ni de crecimiento en el ámbito de redes y telemática. Según los mismos estudiantes, el aprovechamiento de los cursos y su objetivo principal es ganar experiencia en la parte técnica y tratar de trasladarse a otros sectores más centralizados, y una vez instalados en un buen trabajo continuar los estudios para desarrollarse profesionalmente.

Podemos apreciar en el gráfico 7 que iniciaron dieciséis estudiantes, sin embargo solo catorce aprobaron el módulo1, porque una persona desertó y otra no logró aprobar. En el módulo 2 solo aprobaron trece estudiantes y hubo una deserción de tres estudiantes y cuatro no lograron aprobar, llegando al módulo 3 solo seis estudiantes, que se mantuvieron hasta el módulo 4 y se graduaron. 


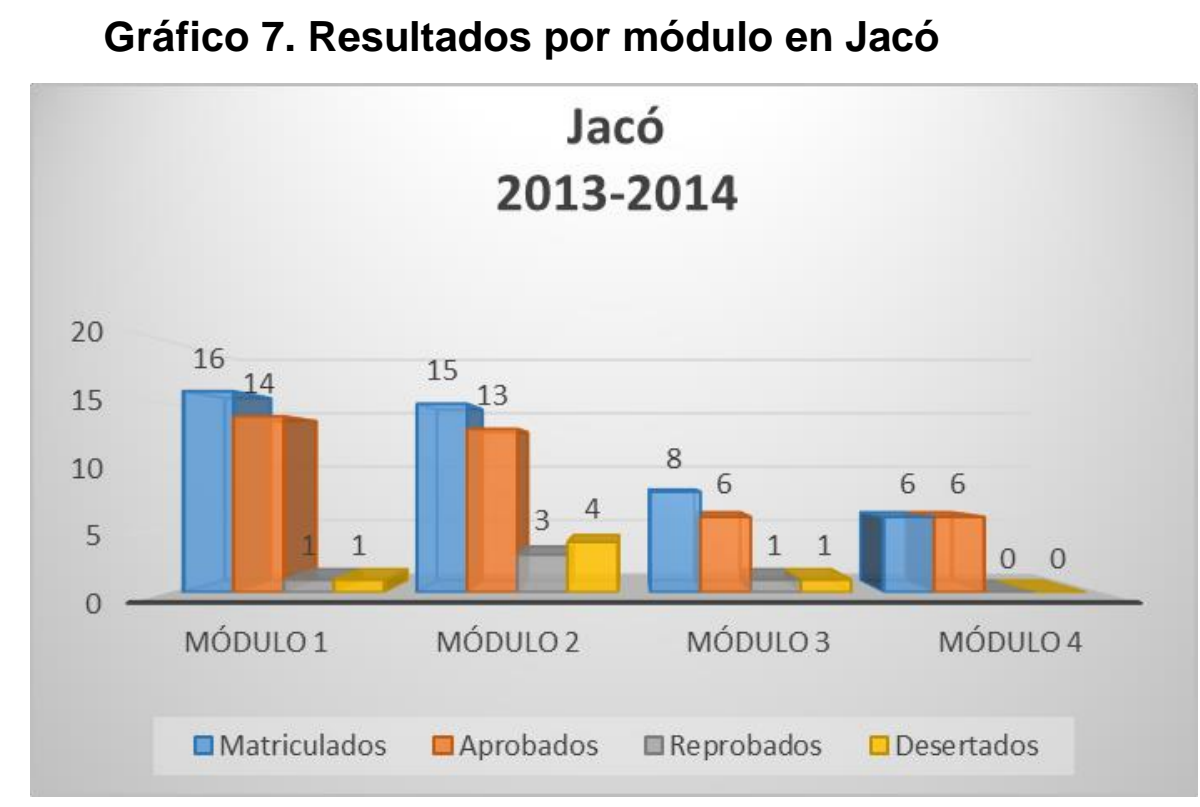

Fuente: Elaboración propia

Gráfico 8. Total de graduados y graduadas

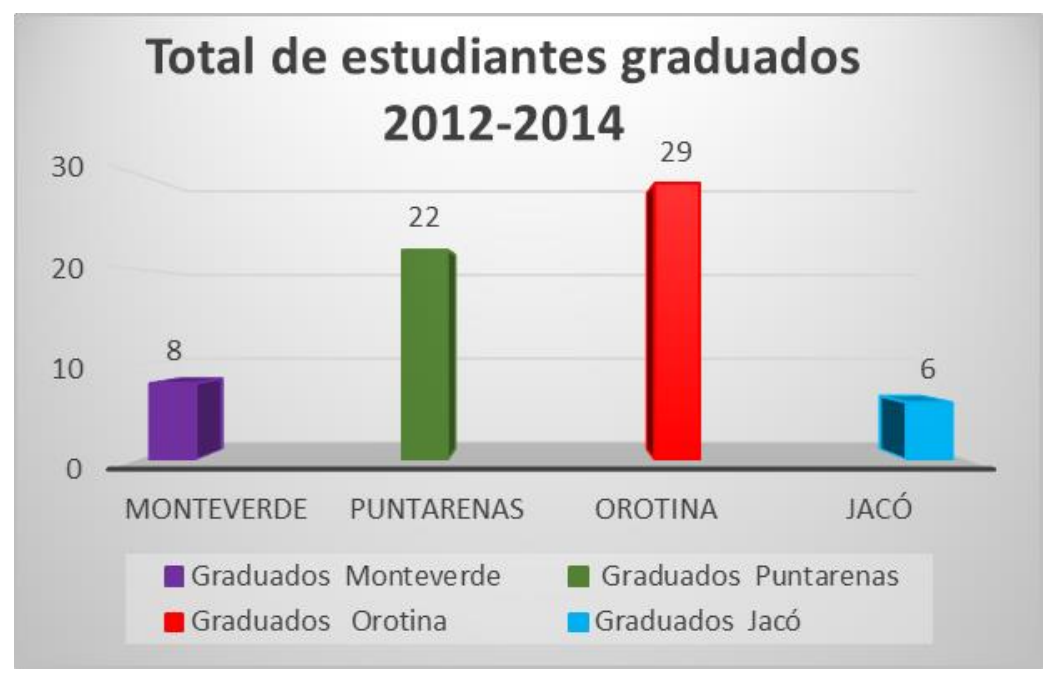

Fuente: Elaboración propia

En el gráfico 8 se muestra que, de un total de 124 estudiantes que iniciaron, 76 lograron graduarse. Las personas interesadas en la iniciativa del Técnico en Telemática y Seguridad Informática (TTSI) se comprometieron y lograron su objetivo. Este proyecto generó un gran interés, reflejado en el impacto que se 
puede apreciar en el total de personas graduadas en las zonas de Orotina, Puntarenas y Quepos. Igualmente, se puede apreciar el impacto de este proyecto porque ha generado un beneficio social en todas las zonas donde se impartió el técnico, ya que todos accedieron a un proyecto que no tuvo ningún costo, fue totalmente gratis, por lo que se concluye que los recursos han sido bien distribuidos para mejorar la calidad de vida de estas personas y así poder tener una mejor opción en el mercado laboral. La inversión de CONARE en estas iniciativas es un incentivo a estas personas de escasos recursos, sin ninguna posibilidad de estudio, que no solo impactó sus vidas, sino las de sus familiares como beneficiados secundarios.

\section{Gráfico 9. Nivel de aprobación}

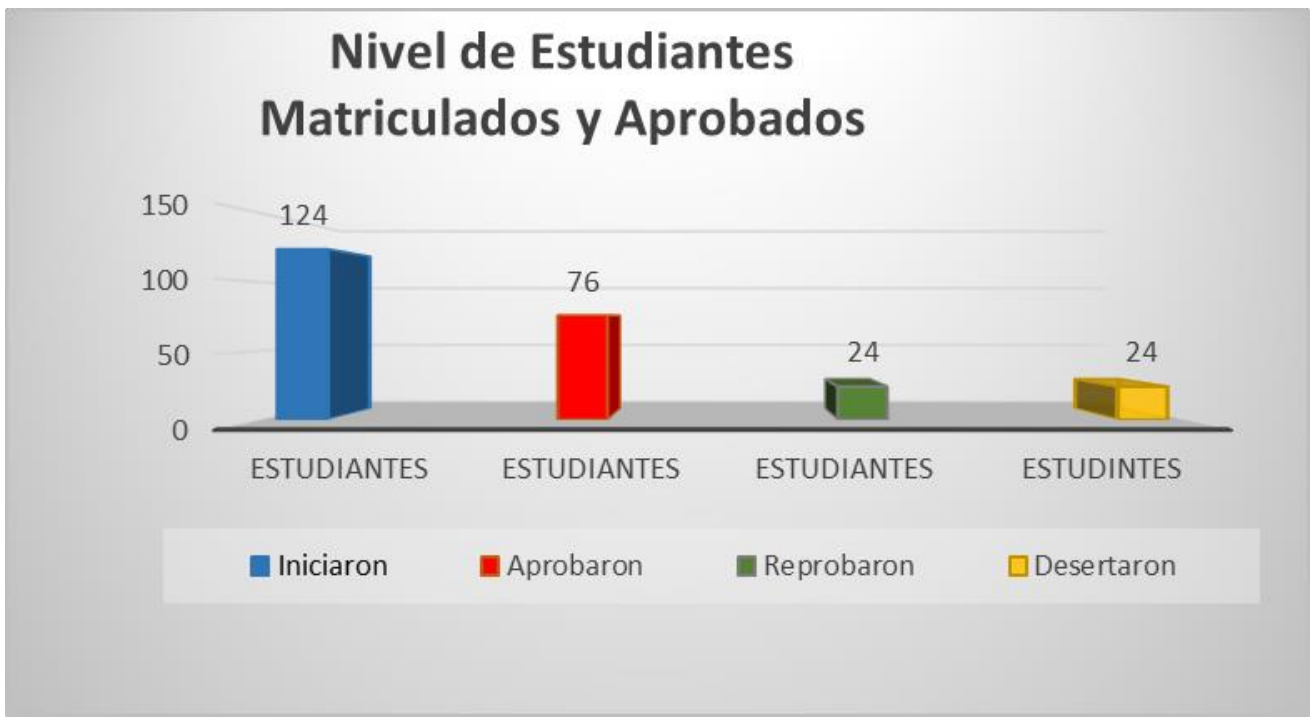

Fuente: Elaboración propia

Se concluye que, de un total de 124 estudiantes que iniciaron, 76 lograron aprobar y culminar el Técnico en Telemática y Seguridad Informática, 24 desertó y 24 no aprobó el programa.

Según se muestra en el gráfico 9, en cuanto a la deserción Jacó fue el lugar donde más se presentó, las razones más fuertes fueron los cambios de horario en los 
trabajos de varios estudiantes. Este es un lugar donde la fuente de trabajo es el turismo y, por otra parte, la emigración de las personas a otros lugares es constante, porque la fuente de trabajo en temporada baja es difícil.

\section{Conclusiones}

EI Técnico en Telemática y Seguridad Informática que la UNED ha estado impartiendo en la zona del Pacífico Central representa una excelente respuesta a las necesidades que enfrentan estas poblaciones para actualizarse en el tema de las TIC y a las necesidades de las empresas de contratar personal especializado. Una vez finalizada la primera etapa del Técnico en Telemática y Seguridad Informática, los y las estudiantes manifestaron un gran interés en realizar cambios en sus vidas. Algunos han tenido nuevas opciones de empleo y ven una oportunidad para enfrentarse no solo al mundo en el ámbito laboral, sino que les da la posibilidad de poner en práctica todo lo aprendido y poder iniciar un proyecto como microempresarios.

El personal académico involucrado en el proyecto ha manifestado que existe un gran interés de parte de todos los estudiantes por continuar los estudios en otras áreas de las TIC y por que la UNED les proporcione una respuesta con la responsabilidad que ha tenido hasta el momento.

En este proyecto se les ha dado seguimiento y acompañamiento a las y los estudiantes, algunos han desarrollado dentro de los mismos cursos recibidos propuestas de proyectos reales que les han permitido llevar a la práctica alguna estrategia de conocimiento en este campo, con criterios técnicos que demuestran los conocimientos, las destrezas y las habilidades que han adquirido en el ámbito de las redes informáticas y otras herramientas tecnológicas que les permiten estar en constante comunicación con sus compañeros para el logro de objetivos planteados, sobre todo porque este es un trabajo en equipo donde deben trabajar con otros estudiantes. 
Como queda demostrado, este proyecto tiene un índice de deserción muy baja. Se muestra un indicador con algunos valores para lograr las metas, como el compromiso, la responsabilidad, las habilidades y las destrezas de cada uno de los estudiantes para salir adelante con el proyecto. Igualmente, otro proceso que ayudó en este sentido fue el haber tomado el tiempo para la escogencia de los perfiles de entrada adecuados. Se hizo una escogencia exhaustiva que duro tres meses para la selección, así como el llenado de un formulario digital.

El programa académico es un proyecto innovador que muestra calidad en la educación no formal que ofrece la universidad, en especial en materia de educación técnica en TIC. Está amparado bajo los currículos de los cursos de CCNA de CISCO que se les imparte a las y los estudiantes como un complemento del Técnico en Telemática y Seguridad Informática; esto contribuye tanto a nivel de conocimiento como a nivel motivacional y de compromiso, ya que abre un camino de oportunidades de mejora en el ámbito laboral y empresarial.

Los y las estudiantes que se graduaron en este programa han desarrollado nuevas oportunidades de formación técnica por medio del conocimiento y de las habilidades que adquirieron, así como la actualización en la generación de nuevas tendencias en las TIC.

Es importante tener claro que en esta experiencia no se pretende dejar a los y las estudiantes con este nivel, sino crear un nuevo grupo para fortalecer las actualizaciones e incrementar los conocimientos bajo los estándares de calidad en esta área, y prepararlos así para las certificaciones internacionales.

Este proyecto ha mantenido a los participantes monitoreados desde dos modalidades: una virtual, por medio de la plataforma institucional y de la academia, y la otra se ha trabajado por medio de la modalidad de visitas de seguimiento, observación en las tutorías presenciales. También se desarrollaron 
algunas evaluaciones para el fortalecimiento del proyecto y se tomaron en cuenta los siguientes indicadores: plan de estudio, materiales didácticos, seguimiento a los y las estudiantes, entre otros. Todo esto permite al programa mantener su nivel de calidad por medio de la retroalimentación para futuras réplicas del proyecto en otras regiones del país.

Se espera que esta exitosa experiencia sea replicada en distintas regiones del país para dar oportunidades de estudio y de mejoramiento en educación no formal a más poblaciones del territorio nacional, y así incentivar los emprendimientos de los y las estudiantes con proyectos innovadores que les permitan dotar a las empresas de las regiones.

En conclusión, el proyecto ha significado un continuo aprendizaje, no solo para los y las estudiantes, sino para toda la unidad académica encargada: coordinación, instructores, centros universitarios y el desarrollo logístico, entre otros involucrados, que en medio de todo el proyecto han trabajado por y para los estudiantes. Igualmente, las evaluaciones nos han retroalimentado con respecto a las debilidades y oportunidades que se presentaron en el camino, con el afán de mejorar y lograr un equilibrio en los diferentes procesos.

Esta es una respuesta a las necesidades que, tanto internacionalmente como en el país, las empresas y organizaciones como CINDE y la Cámara de empresarios en TIC (CAMTIC) han expresado en múltiples espacios sociales y que las universidades deben ofrecer cada vez más a las poblaciones, tanto la profesional para su actualización como la no profesional para ofrecer una alternativa importante de obtención de empleo o desarrollo propio. 


\section{Recomendaciones}

Dada la retroalimentación que los y las estudiantes han ofrecido al Técnico en Telemática y Seguridad Informática, es imprescindible continuar con el programa a fin de que otras personas se vean favorecidas con la formación técnica que les permita el desarrollo personal y regional.

La UNED debe promover con mayor fuerza la educación continua técnica que permita impactar a las nuevas generaciones que se encuentran en situación de desventaja, tanto para promover el emprendedurismo como para abrir posibilidades de trabajo en los centros transnacionales de bajo costo (HP, INTEL, IBM, Amazon, entre otros).

Debido al impacto proyectado en Orotina con la construcción del aeropuerto, promovido por el Gobierno como proyecto país, es importante que la población de esta región cuente con programas de educación técnica que le permitan ser competitiva y tener la oportunidad de ocupar puestos en empresas nacionales y transnacionales antes de que sean ocupados por externos a la región.

La oferta de este programa técnico en el caso de Orotina puede promover la disminución de desempleo que ha generado la ruta 27 , lo que ha afectado a los comercios y a las pequeñas y medianas empresas, ya que el turismo se mueve en otra dirección, por lo que se recomienda su continuidad.

En las otras regiones impactadas con este programa de formación técnica (Quepos, Monteverde y Puntarenas), al ser zonas turísticas, se ha permitido el desarrollo de emprendimientos en TIC, por lo que la UNED debe promover la actualización permanente de este tipo de programas. 
En congruencia con los planes de CONARE, se recomienda replicar estos proyectos de formación técnica, no solo en la región Pacífico Central, sino en las otras regiones del país.

\section{Referencias}

Estado de la Nación. (2003). Educación Técnica y Formación profesional en Costa Rica "Avances y Desafíos". Informe del Estado de la Nación, 237-238.

Estado de la Nación (2003). Educación Técnica y Formación Profesional en Costa Rica: Avances y Desafíos (introducción (Interuniversitaria) (interuniversitaria, 2014)n). Recuperado de http://www.estadonacion.or.cr/files/biblioteca_virtual/educacion/003/parte_1 _capitulo_5-edu03.pdf

Programa de Regionalización Interuniversitaria de CONARE. (2012-2014) Iniciativas Interuniversitaria Regional del Pacífico Central "Proyecto de Innovación y Gestión en TIC, cuarta Publicación. Recuperado de http://www.conare.ac.cr/iniciativas-interuniversitarias

Programa de Regionalización Interuniversitaria de CONARE. (2014). Informe semestral. San José, Costa Rica: UNED. 